\title{
Thermal Conductivity of Composite Materials Containing Copper Nanowires
}

\author{
Dahai Zhu, ${ }^{1}$ Wei Yu, ${ }^{1}$ Haixu Du, ${ }^{2}$ Lifei Chen, ${ }^{1}$ Yang Li, ${ }^{1}$ and Huaqing Xie ${ }^{1}$ \\ ${ }^{1}$ College of Engineering, Shanghai Polytechnic University, Shanghai 201209, China \\ ${ }^{2}$ College of Materials Science and Engineering, Donghua University, Shanghai 201620, China \\ Correspondence should be addressed to Wei Yu; yuwei@sspu.edu.cn
}

Received 9 May 2016; Accepted 19 September 2016

Academic Editor: Stefano Bellucci

Copyright (c) 2016 Dahai Zhu et al. This is an open access article distributed under the Creative Commons Attribution License, which permits unrestricted use, distribution, and reproduction in any medium, provided the original work is properly cited.

\begin{abstract}
The development of thermal conductive polymer composite is necessary for the application in thermal management. In this paper, the experimental and theoretical investigations have been conducted to determine the effect of copper nanowires (CuNWs) and copper nanoparticles (CuNPs) on the thermal conductivity of dimethicone nanocomposites. The CuNWs and CuNPs were prepared by using a liquid phase reduction method, and they were characterized through scanning electron microscopy (SEM) and Xray diffraction (XRD). The experimental data show that the thermal conductivity of composites increases with the increase of filler. With the addition of $10 \mathrm{vol} . \%$ CuNWs, the thermal conductivity of the composite is $0.41 \mathrm{~W} / \mathrm{m} / \mathrm{K}$. The normalized thermal conductivity enhancement factor is 2.73 , much higher than that of the analogue containing CuNPs (1.67). These experimental data are in agreement with Nan's model prediction. Due to the high aspect ratio of 1D CuNWs, they can construct thermal networks more effectively than CuNPs in the composite, resulting in higher thermal conductivity.
\end{abstract}

\section{Introduction}

Due to the unique physicochemical properties, lightweight, and cost-effective process ability, polymer nanocomposites have attracted considerable interest, such as increased electrical/thermal conductivity and enhanced mechanical properties (stiffness and strength) [1]. In recent years, a burst of researches has been witnessed in the incorporation of metal nanoparticles into a polymer matrix. This interest arises in polymer composites since they can preserve the mechanical properties of the polymer matrix but can benefit in heat conduction properties due to the inclusion of metal fillers [2]. Among the various nanosized metal particles, gold and silver were frequently used as nanofillers for polymer matrices due to their good conductivity and chemical stability. It is likely to improve thermal and other properties of the host polymer [37]. Generally speaking, the high cost of these nanomaterials precludes the extensive use in industrial fields. Therefore, there is an urgent need for alternative metal nanofillers, which have appropriate thermal conductive property with a much lower cost than gold and silver.
The most important reason for using copper in thermal applications is that it has the highest conductivity of metals at room temperature apart from silver, and it happens to be more abundant/cheaper than silver [8]. At the same time, it has high electrical conductivity, high thermal conductivity, and easy fabrication process; therefore, this leads to potential application in electron conduction slurry, microelectronics, and so on [9]. Metallic copper nanocrystals are mainly two types of nanostructures: copper nanowires (CuNWs) and copper nanoparticles (CuNPs). CuNWs are the onedimensional (1D) nanostructures, and CuNPs are zerodimensional (OD) nanomaterials. It is well known that the original intrinsic properties of metal nanostructures are largely determined by their size, structure, and mutual interaction between nanoparticles [10]. Compared with nanoparticles, 1D nanowires have smaller dimension structure and high aspect ratio, which could efficiently transport electrical and thermal carriers along one controllable direction [1113]. Therefore, intensive researches have been ongoing for synthesis of one-dimensional copper nanostructures with controlled size and shape [14-21]. 
Copper nanostructure materials have been used in different matrices to improve the electrical and thermal properties. Rathmell et al. [21] prepared CuNW/PVDF nanocomposites with a precipitation technique followed by compression molding. It was observed that CuNW/PVDF had higher dielectric permittivity and lower dielectric loss than the MWCNT/PVDF nanocomposites at room temperature. Copper nanoparticles were usually utilized for improving the thermal properties of nanofluid with different base fluid [22-25]. Zeng et al. [26] investigated the effect of copper nanowires with ultrahigh aspect ratio and sponge-like structure on the properties of organic phase change materials, and the thermal conductivity of the composite enhanced greatly compared with base materials. Wang et al. [27] reported thermal interface materials with high-aspect-ratio CuNWs, which showed low percolation threshold and high thermal conductivity with ultralow volume loading.

Because copper nanostructures are easily oxidized in ambient air, the widely used method for the preparation of copper nanocrystals is the liquid phase chemical reduction method with capping agents. It can protect copper nanoparticles against oxidation and agglomeration. In this work, we prepared copper nanowires (CuNWs) and copper nanoparticles (CuNPs) through liquid phase reduction method and investigated the influence of content and shape on the thermal conductivity of the dimethicone nanocomposites. It is very interesting that $1 \mathrm{D}$ CuNWs can construct thermal networks more effectively than $\mathrm{Cu}$ nanoparticles due to the high aspect ratio of CuNWs.

\section{Experimental Details}

2.1. Synthesis of CuNWs. The CuNWs were prepared by the reduction of copper nitrate $\left(\mathrm{Cu}\left(\mathrm{NO}_{3}\right)_{2}\right)$ through hydrazine in an aqueous solution containing sodium hydroxide $(\mathrm{NaOH})$ and ethylenediamine (EDA). CuNWs were prepared by three steps [16]. Firstly, $\mathrm{Cu}\left(\mathrm{NO}_{3}\right)_{2}(100 \mathrm{~mL}, 0.2 \mathrm{M})$ was added slowly with stirring to $\mathrm{NaOH}$ aqueous solution $(1200 \mathrm{~mL}$, $25 \mathrm{M})$. Then, EDA $(30 \mathrm{~mL})$ and hydrazine $(2.5 \mathrm{~mL}, 35 \mathrm{wt} \%)$ were slowly added to the above solution followed by vigorous mixing for $1 \mathrm{~h}$ by magnetic stirrer at $80^{\circ} \mathrm{C}$ until a reddish brown solution was obtained. After the reaction, the resulting reddish brown solution was pumped off with vacuum in filtering and then washed with a $3 \mathrm{wt} \%$ aqueous solution of hydrazine and stored in the same hydrazine solution at room temperature under an argon atmosphere to minimize oxidation.

2.2. Synthesis of CuNPs. Copper nanoparticles were synthesized in aqueous solution by reducing copper (II) sulfate pentahydrate $\left(\mathrm{CuSO}_{4} \cdot 5 \mathrm{H}_{2} \mathrm{O}\right)$ with hydrazine containing poly $(\mathrm{N}$ vinylpyrrolidone) (PVP) and diethylene-glycol (DEG) [28]. The detailed synthetic procedure of copper nanoparticles was as follows: PVP, as a protecting agent, was firstly dissolved in DEG. The aqueous solution of $\mathrm{CuSO}_{4} \cdot 5 \mathrm{H}_{2} \mathrm{O}$ was added to the DEG solution and the solution was heated to reaction temperatures. Hydrazine, used as a reducing agent, was added to the hot reaction medium drop by drop. After $1 \mathrm{~h}$ of reaction, the solution was cooled to room temperature and filtered and then washed with deionized water and ethanol sequentially. The process was repeated several times to remove the excess surfactant, and a reddish fluffy solid was obtained. The product was dried at $80^{\circ} \mathrm{C}$ in an inert nitrogen atmosphere.

2.3. Preparation of Nanocomposites. The nanocomposites were prepared as follows: The CuNWs with different volume fraction were mixed with dimethicone by mechanical grinding in a mortar for $15 \mathrm{~min}$. The total process of CuNWs based dimethicone nanocomposite was illustrated in Figure 1. For comparison, the analogue containing CuNPs was also prepared by the same method.

2.4. Characterization. Scanning electron microscopy (SEM, Hitachi S4800) was used to examine the dimension and shape of CuNWs and CuNPs. Phase composition and crystallinity of CuNWs and CuNPs were recorded using X-ray diffractometer (XRD, D8-Advance, Germany) with a back monochromator operating at $40 \mathrm{kV}$ and a copper cathode as the X-ray source $(\lambda=0.154 \mathrm{~nm})$. XRD patterns were recorded from 20 to $80^{\circ}(2 \theta)$ with a scanning step of $0.01^{\circ}$. A thermal conductivity analyzer from $\mathrm{Tci}^{\mathrm{TM}} / \mathrm{C}$-Therm was used to measure thermal conductivity of the dimethicone nanocomposites. This instrument is a state-of-theart thermal property characterization instrument based on the modified transient plane source (TPS) technique. The TCi system consists of a sensor, power control device, and computer software. A spiral-type heating source is located at the center of the sensor, and heat is generated at the center. The heat that has been generated enters the material through the sensor during which a voltage decrease occurs rapidly at the heating source, and the thermal conductivity is calculated through the voltage decrease data. The testing capabilities of the system are 0 to $100 \mathrm{~W} / \mathrm{m} / \mathrm{K}$ across a wide range of temperature $\left(-50\right.$ to $200^{\circ} \mathrm{C}$ ). PDMS (dimethyl polysiloxane silicone fluid) is provided as a standard reference material with the TCi thermal conductivity analyzer, and the accuracy of these measurements was estimated to be within $\pm 1 \%$. For this measurement, the samples were filled into the mould with a thickness of $2 \mathrm{~mm}$. The thermal conductivity of each sample is tested 5 times to compute an average value. The temperature of test system was controlled at $25^{\circ} \mathrm{C}$ by constant temperature box (Shanghai Boxun Industry \& Commerce Co., Ltd.).

\section{Results and Discussion}

In theory, the composites containing $1 \mathrm{D}$ nanowires have higher thermal conductivity than 0D nanoparticles, but there are seldom these researches because the preparation method for $1 \mathrm{D}$ CuNWs is very complex. But now, Chang et al. [16] found the method to synthesize high-quality ultralong copper nanowires in large scale. It gives us the chance to verify the characterizations.

Figure 2 shows the typical SEM images of CuNWs and CuNPs. Seen from Figure 2(b), the as-synthesized CuNWs display wire-like structure with a uniform diameter of 


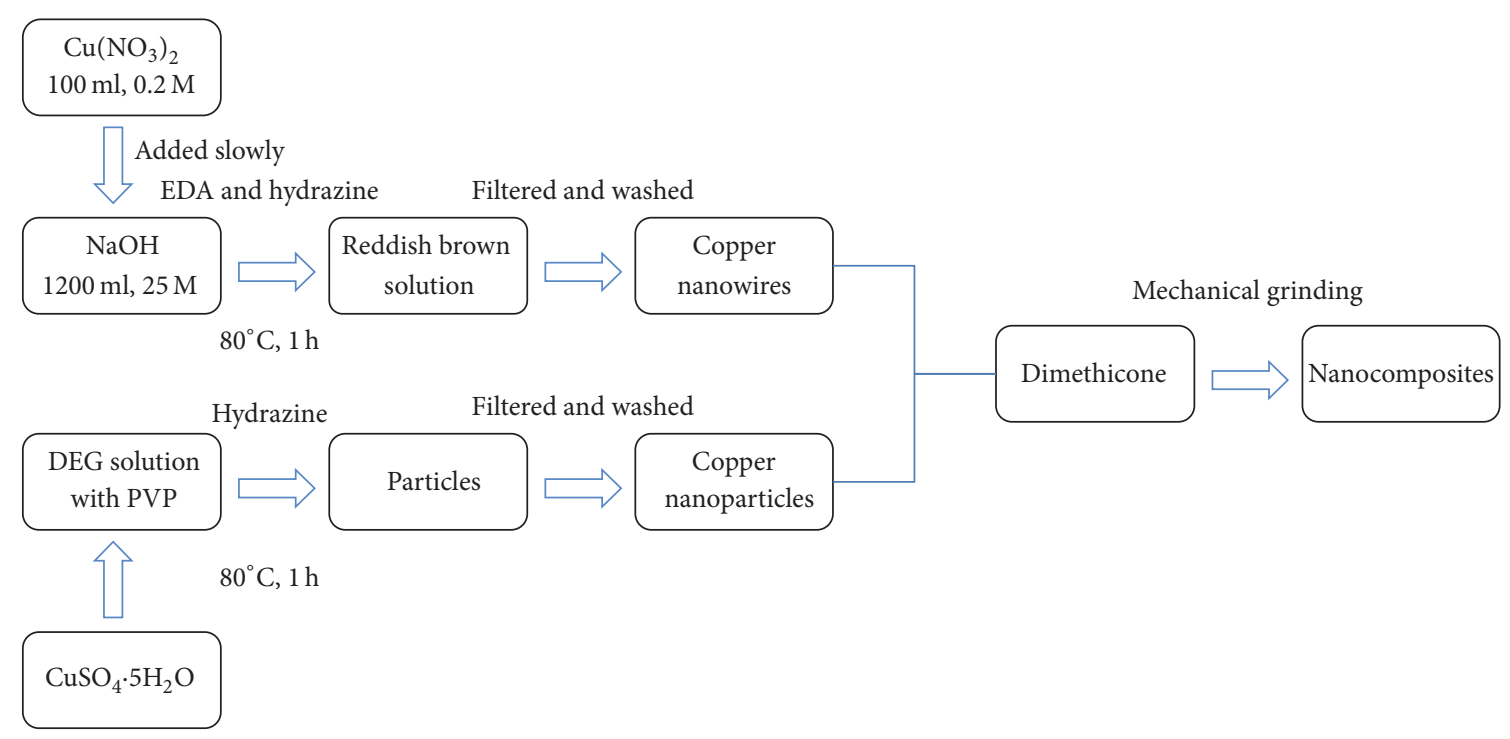

FIGURE 1: The preparation process for dimethicone nanocomposites.

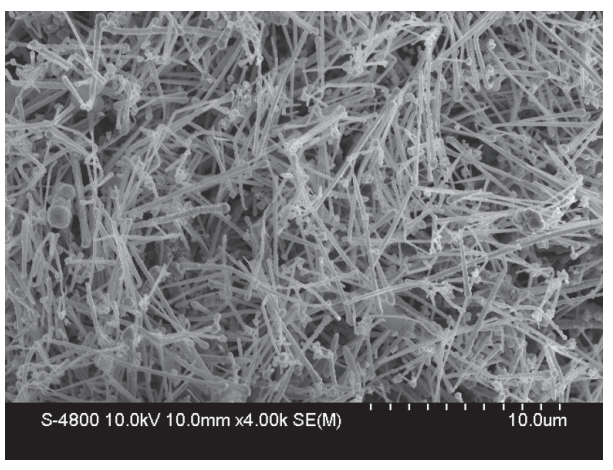

(a)

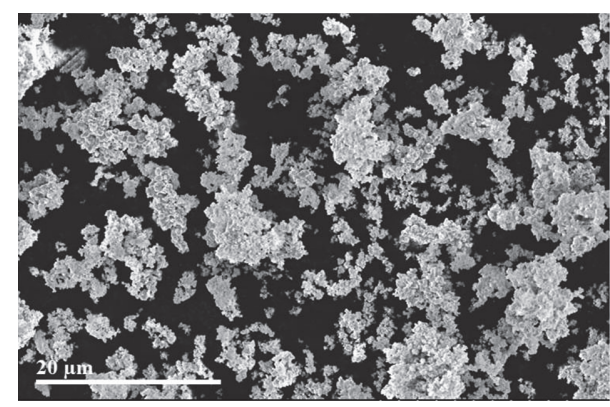

(c)

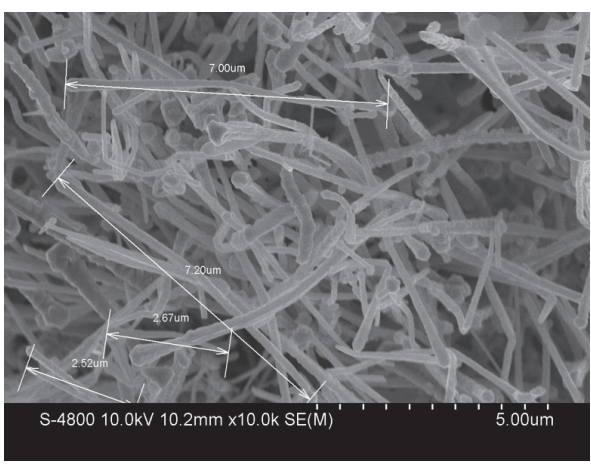

(b)

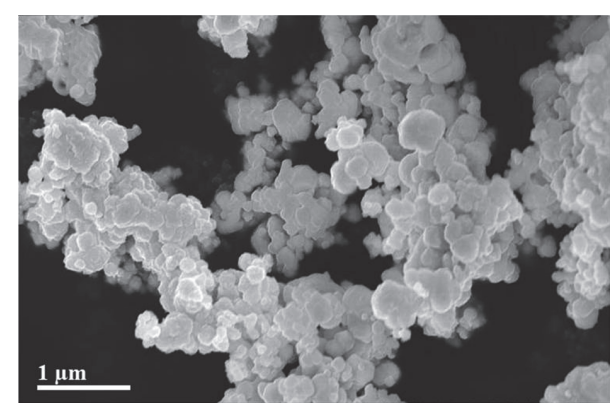

(d)

FIgure 2: The typical SEM images of (a) CuNWs and (c) CuNPs at low magnification and (b) CuNWs and (d) CuNPs at high magnification.

$200 \mathrm{~nm}$, and the length varies from 2 to 7 micrometers. Besides, the nanowires are found to be highly flexible as some of them showed bending more than $180^{\circ}$ without any fracture. These results imply that the CuNWs with high aspect ratio are synthesized successfully. We can also observe spherical nanoparticles attached at one end of nanowires, indicating that the CuNWs grew from spherical seeds, which is revealed by Rathmell et al. [21]. Based on their report, the CuNWs grow via atomic addition to $\{110\}$ planes, which have the highest-surface energy among the low-index facets of copper [21]. Meanwhile, the addition of EDA to the reaction solution is also necessary to promote anisotropic growth of CuNWs. Suitable addition of EDA binds preferentially to the side facets of the CuNWs; this could cause preferential growth along the axial $\{110\}$ direction [21]. For the CuNPs, shown in Figure 2(d), it can be seen that the product consists of spherical particles. The diameter of nanoparticles ranges from 50 to $100 \mathrm{~nm}$. 


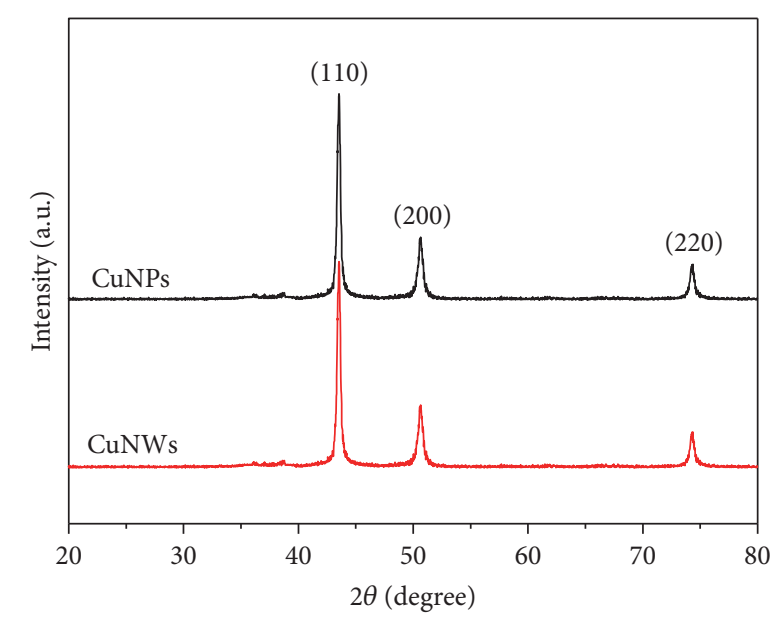

Figure 3: The XRD patterns of CuNWs and CuNPs.

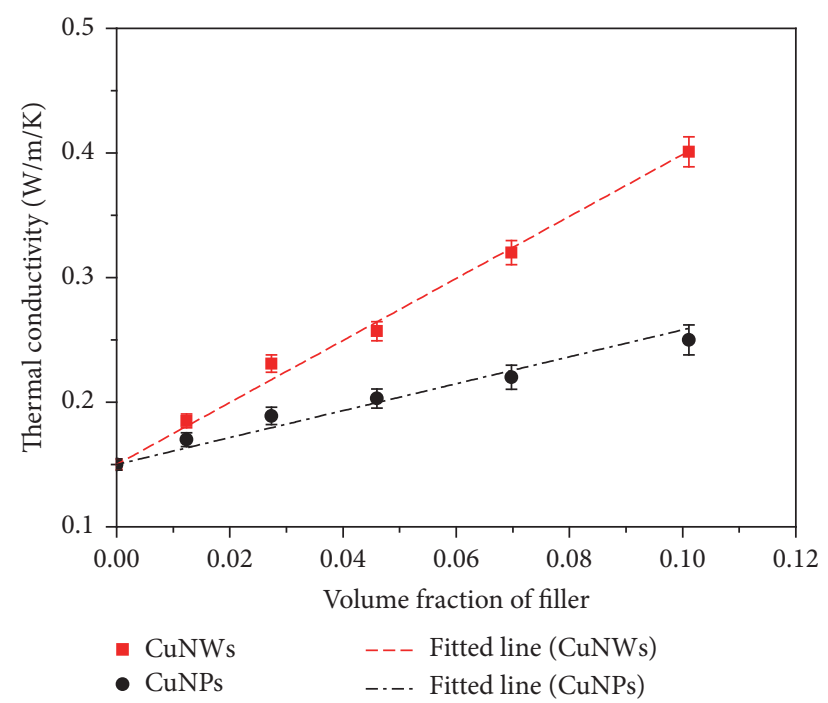

FIGURE 4: Comparison of theoretical predictive model with experimental data.

Figure 3 is the XRD pattern of CuNWs and CuNPs. It is clear that the relative intensity and the $2 \theta$ of diffraction peaks of CuNWs and CuNPs spectra are identical. The peaks correspond to the $\{110\},\{200\}$, and $\{220\}$ crystal planes of face-centered cubic copper (JCPDS \# 03-1018), respectively [29]. There is no diffraction peak of copper oxide or other crystalline materials existed, indicating that the CuNWs and the CuNPs are all in the form of pure copper metal.

The measured experimental values of the thermal conductivity of dimethicone nanocomposites containing CuNWs and CuNPs are shown in Figure 4. The maximum loading level is $10 \mathrm{vol} . \%$, because beyond this value the fillers cannot add to the dimethicone the matrix to prepare uniform and stable composites. Seen from Figure 4, the thermal conductivity of dimethicone is very low, only $0.15 \mathrm{~W} / \mathrm{m} / \mathrm{K}$. Because of the high thermal conductivity of the filler, the thermal conductivity of the composites increases with the increase of both CuNWs and CuNPs loading. It can also be

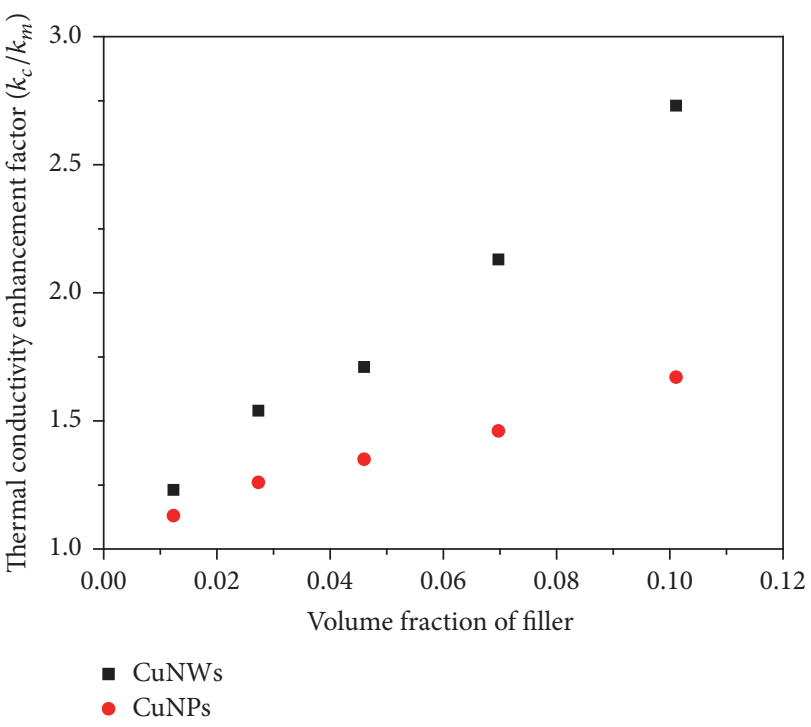

FIGURE 5: Thermal conductivity enhancement factor of different dimethicone nanocomposites as a function of loading.

seen that the thermal conductivity of the composites depends on the size and shape of $\mathrm{Cu}$ particles. The thermal conductivities of dimethicone composites containing $10 \mathrm{vol} \%$ of CuNPs and CuNWs are $0.25 \mathrm{~W} / \mathrm{m} / \mathrm{K}$ and $0.41 \mathrm{~W} / \mathrm{m} / \mathrm{K}$, respectively. It indicates that the CuNWs' ability to enhance thermal conductivity is higher than CuNPs. There is a widespread view among composite materials containing nanomaterials as thermal conductive filler proved by Figure 4, in which no percolation threshold can be observed. Seen from Figure 5 , the normalized thermal conductivity enhancement factors $\left(k_{c} / k_{m}\right)$ of dimethicone nanocomposites with CuNWs increase substantially with the increase of the volume fraction. The maximum is up to 2.73 with 10 vol.\% CuNWs addition, much higher than that of dimethicone nanocomposites with the same CuNPs addition (1.67).

To further investigate the improvement of the thermal conductivity of nanocomposites with CuNWs and CuNPs, the experimental results are compared with the theoretical values predicted by Nan's model equation [30, 31]:

$$
\frac{k_{c}}{k_{m}}=1+\frac{\varphi p}{3} \frac{k_{f} / k_{m}}{p+2 a_{K} k_{f} / d k_{m}},
$$

where $\varphi$ is the volume fraction of the fillers, $k_{c}$ is the thermal conductivity of the composite, $k_{m}$ is the thermal conductivity of the dimethicone $(0.15 \mathrm{~W} / \mathrm{m} / \mathrm{K}), k_{f}$ is the thermal conductivity of filler $(400 \mathrm{~W} / \mathrm{m} / \mathrm{K}), a_{K}$ is the Kapitza radius $\left(R_{k} \times k_{m}\right.$, where $R_{k}$ is interface thermal resistance between filler and matrix), $p$ is the aspect ratio of the filler, and $d$ is the diameter of the filler.

The theoretical thermal conductivity value is presented in Figure 4. Using a numerical best fit of the data to the model, $R_{k}$ is set to $3.3 \times 10^{-7} \mathrm{Km}^{2} / \mathrm{W}$, for fitted line (CuNWs), $p$ is the aspect of CuNWs ( 25), and, for fitted line (CuNPs), $p$ is the aspect of CuNPs $(\sim 1)$. The experimental data of the thermal conductivity of composites with CuNWs and 


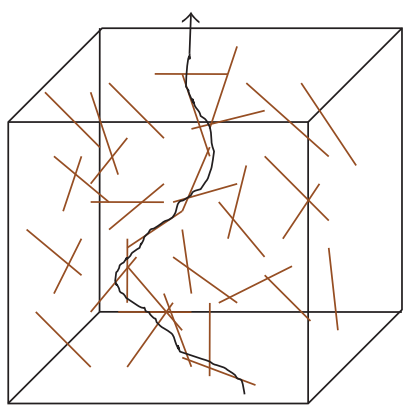

- CuNWs

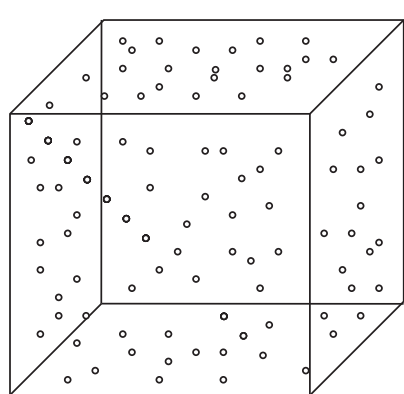

$\circ$ CuNPs (a)

(b)

Figure 6: Schematics of the distribution of (a) CuNWs and (b) CuNPs in dimethicone base.

CuNPs are in reasonable agreement with the predicted results of Nan's equation (CuNWs) and Nan's equation (CuNPs), respectively. It indicates that the shape and size of filler have a great influence on thermal conductivity of the composites, and CuNWs are superior as filler for dimethicone composite compared with CuNPs.

To better demonstrate the superiority of CuNWs, the thermal conduction model of metal-dimethicone composites was proposed, as illustrated in Figure 6. Polymer usually has low thermal conductivity due to the low density, low sound speed, and molecular disorder of the polymer that greatly suppresses phonon transport. The introduced thermal conductive particles could help to improve the thermal conductive property; however, the outcomes are not always desirable owing to the interfacial thermal resistance. The thermal conductivity of the nanocomposite with CuNWs, described in experimental values and theoretical prediction, is higher than that of CuNPs. The main reason, shown in Figure 6(a), is that the CuNWs used in this research with a large aspect ratio can easily form bridges between themselves and thus construct some effective thermal conductive networks. These networks provide a low-resistance pathway to heat conduction and increase the overall thermal conductivity of the composite [27, 32]. Nevertheless, the CuNPs are very small and surrounded or encapsulated by polymer matrix, shown in Figure 6(b). It makes them unable to touch each other to form effective thermal conductive pathways in this composite, resulting in the lower thermal conductivity. We also compared the composites' thermal transportation properties containing copper nanowire, graphite, and graphene nanoplatelets (GNPs) [33]. It is found that GNPs are the best, then CuNPs, while graphite is the poorest. From the above analysis, we can get the composites with higher thermal conductivity through the shape control of nanoparticles, especially for the high-aspect-ratio 1D or 2D nanomaterials.

\section{Conclusions}

In this work, we report an improvement of the thermal conductivity of dimethicone composites containing nanostructured copper. And the different size and shape of nanostructured copper exhibit different influence on thermophysical property of the nanocomposites. The thermal conductivity of the dimethicone nanocomposites increases with increase of nanostructured copper content and the experimental data is in reasonable agreement with Nan's model prediction. The thermal conductivity of the dimethicone composite filled with CuNWs is higher than that of CuNPs under the same filler content, which is due to construction of effective thermal networks in the composite with CuNWs.

\section{Competing Interests}

The authors declare that they have no competing interests.

\section{Acknowledgments}

The work was supported by National Natural Science Foundation of China (51476094, 51590901), Shu Guang project supported by Shanghai Municipal Education Commission and Shanghai Education Development Foundation (15SG52), and the key subject of Shanghai Polytechnic University (Material Science and Engineering, XXKZD1601).

\section{References}

[1] H. Althues, J. Henle, and S. Kaskel, "Functional inorganic nanofillers for transparent polymers," Chemical Society Reviews, vol. 36, no. 9, pp. 1454-1465, 2007.

[2] V. E. Gul and L. Z. Shenfill, Conductive Polymer Composites, Khimia, Moscow, Russia, 1984.

[3] P. Calvert, "Inkjet printing for materials and devices," Chemistry of Materials, vol. 13, no. 10, pp. 3299-3305, 2001.

[4] K. Woo, D. Kim, J. S. Kim, S. Lim, and J. Moon, "Ink-jet printing of $\mathrm{Cu}$-Ag-based highly conductive tracks on a transparent substrate," Langmuir, vol. 25, no. 1, pp. 429-433, 2009.

[5] L. M. Davis and D. W. Thompson, "Novel and facile approach to the fabrication of metal-patterned dielectric substrates," Chemistry of Materials, vol. 19, no. 9, pp. 2299-2303, 2007.

[6] Y. H. Kim, D. K. Lee, H. G. Cha, C. W. Kim, Y. C. Kang, and Y. S. Kang, "Preparation and characterization of the antibacterial $\mathrm{Cu}$ nanoparticle formed on the surface of $\mathrm{SiO}_{2}$ nanoparticles," Journal of Physical Chemistry B, vol. 110, no. 49, pp. $24923-$ 24928, 2006.

[7] N. Balachander, I. Seshadri, R. J. Mehta et al., "Nanowirefilled polymer composites with ultrahigh thermal conductivity," Applied Physics Letters, vol. 102, no. 9, Article ID 093117, 2013.

[8] U.S. Geological Survey, Mineral Commodity Summaries. Copper. 50, U.S. Geological Survey, 2009.

[9] J. R. Groza and J. C. Gibeling, "Principles of particle selection for dispersion-strengthened copper," Materials Science and Engineering A, vol. 171, no. 1-2, pp. 115-125, 1993.

[10] G. A. Somorjai, F. Tao, and J. Y. Park, "The nanoscience revolution: merging of colloid science, catalysis and nanoelectronics," Topics in Catalysis, vol. 47, no. 1-2, pp. 1-14, 2008.

[11] J. Hu, T. W. Odom, and C. M. Lieber, "Chemistry and physics in one dimension: synthesis and properties of nanowires and nanotubes," Accounts of Chemical Research, vol. 32, no. 5, pp. 435-445, 1999. 
[12] Z. L. Wang, Z. Dai, and S. Sun, "Polyhedral shapes of cobalt nanocrystals and their effect on ordered nanocrystal assembly," Advanced Materials, vol. 12, no. 24, pp. 1944-1946, 2000.

[13] Y. Xia, P. Yang, Y. Sun et al., "One-dimensional nanostructures: synthesis, characterization, and applications," Advanced Materials, vol. 15, no. 5, pp. 353-389, 2003.

[14] Z. Liu and Y. Bando, "A novel method for preparing copper nanorods and nanowires," Advanced Materials, vol. 15, no. 4, pp. 303-305, 2003.

[15] A. A. Athawale, P. P. Katre, M. Kumar, and M. B. Majumdar, "Synthesis of CTAB-IPA reduced copper nanoparticles," Materials Chemistry and Physics, vol. 91, no. 2-3, pp. 507-512, 2005.

[16] Y. Chang, M. L. Lye, and H. C. Zeng, "Large-scale synthesis of high-quality ultralong copper nanowires," Langmuir, vol. 21, no. 9, pp. 3746-3748, 2005.

[17] N. J. Gerein and J. A. Haber, "Effect of ac electrodeposition conditions on the growth of high aspect ratio copper nanowires in porous aluminum oxide templates," Journal of Physical Chemistry B, vol. 109, no. 37, pp. 17372-17385, 2005.

[18] X. Su, J. Zhao, H. Bala et al., "Fast synthesis of stable cubic copper nanocages in the aqueous phase," Journal of Physical Chemistry C, vol. 111, no. 40, pp. 14689-14693, 2007.

[19] P. Kanninen, C. Johans, J. Merta, and K. Kontturi, "Influence of ligand structure on the stability and oxidation of copper nanoparticles," Journal of Colloid and Interface Science, vol. 318, no. 1, pp. 88-95, 2008.

[20] A. Sarkar, T. Mukherjee, and S. Kapoor, "PVP-stabilized copper nanoparticles: a reusable catalyst for 'click' reaction between terminal alkynes and azides in nonaqueous solvents," Journal of Physical Chemistry C, vol. 112, no. 9, pp. 3334-3340, 2008.

[21] A. R. Rathmell, S. M. Bergin, Y.-L. Hua, Z.-Y. Li, and B. J. Wiley, "The growth mechanism of copper nanowires and their properties in flexible, transparent conducting films," Advanced Materials, vol. 22, no. 32, pp. 3558-3563, 2010.

[22] M.-S. Liu, M. C.-C. Lin, C. Y. Tsai, and C.-C. Wang, "Enhancement of thermal conductivity with $\mathrm{Cu}$ for nanofluids using chemical reduction method," International Journal of Heat and Mass Transfer, vol. 49, no. 17-18, pp. 3028-3033, 2006.

[23] W. Yu, H. Xie, L. Chen, and Y. Li, "Investigation on the thermal transport properties of ethylene glycol-based nanofluids containing copper nanoparticles," Powder Technology, vol. 197, no. 3, pp. 218-221, 2010.

[24] U. S. Shenoy and A. N. Shetty, "Simple glucose reduction route for one-step synthesis of copper nanofluids," Applied Nanoscience, vol. 4, no. 1, pp. 47-54, 2014.

[25] N. Nikkam, M. Ghanbarpour, M. Saleemi et al., "Experimental investigation on thermo-physical properties of copper/diethylene glycol nanofluids fabricated via microwaveassisted route," Applied Thermal Engineering, vol. 65, no. 1-2, pp. 158-165, 2014.

[26] J. L. Zeng, F. R. Zhu, S. B. Yu et al., "Effects of copper nanowires on the properties of an organic phase change material," Solar Energy Materials and Solar Cells, vol. 105, pp. 174-178, 2012.

[27] S. Wang, Y. Cheng, R. Wang, J. Sun, and L. Gao, "Highly thermal conductive copper nanowire composites with ultralow loading: toward applications as thermal interface materials," ACS Applied Materials \& Interfaces, vol. 6, no. 9, pp. 6481-6486, 2014.

[28] H. Xie, W. Yu, Y. Li, and L. Chen, "Discussion on the thermal conductivity enhancement of nanofluids," Nanoscale Research Letters, vol. 6, no. 1, article 124, 2011.
[29] Z. Liu, Y. Yang, J. Liang et al., "Synthesis of copper nanowires via a complex-surfactant-assisted hydrothermal reduction process," Journal of Physical Chemistry B, vol. 107, no. 46, pp. 1265812661, 2003.

[30] C.-W. Nan, Z. Shi, and Y. Lin, "A simple model for thermal conductivity of carbon nanotube-based composites," Chemical Physics Letters, vol. 375, no. 5-6, pp. 666-669, 2003.

[31] P. Zhang, Q. Li, and Y. Xuan, "Thermal contact resistance of epoxy composites incorporated with nano-copper particles and the multi-walled carbon nanotubes," Composites Part A: Applied Science and Manufacturing, vol. 57, pp. 1-7, 2014.

[32] W. Jiajun and Y. Xiao-Su, "Effects of interfacial thermal barrier resistance and particle shape and size on the thermal conductivity of AlN/PI composites," Composites Science and Technology, vol. 64, no. 10-11, pp. 1623-1628, 2004.

[33] W. Yu, H. Xie, L. Chen, Z. Zhu, J. Zhao, and Z. Zhang, "Graphene based silicone thermal greases," Physics Letters, Section A: General, Atomic and Solid State Physics, vol. 378, no. 3, pp. 207-211, 2014. 

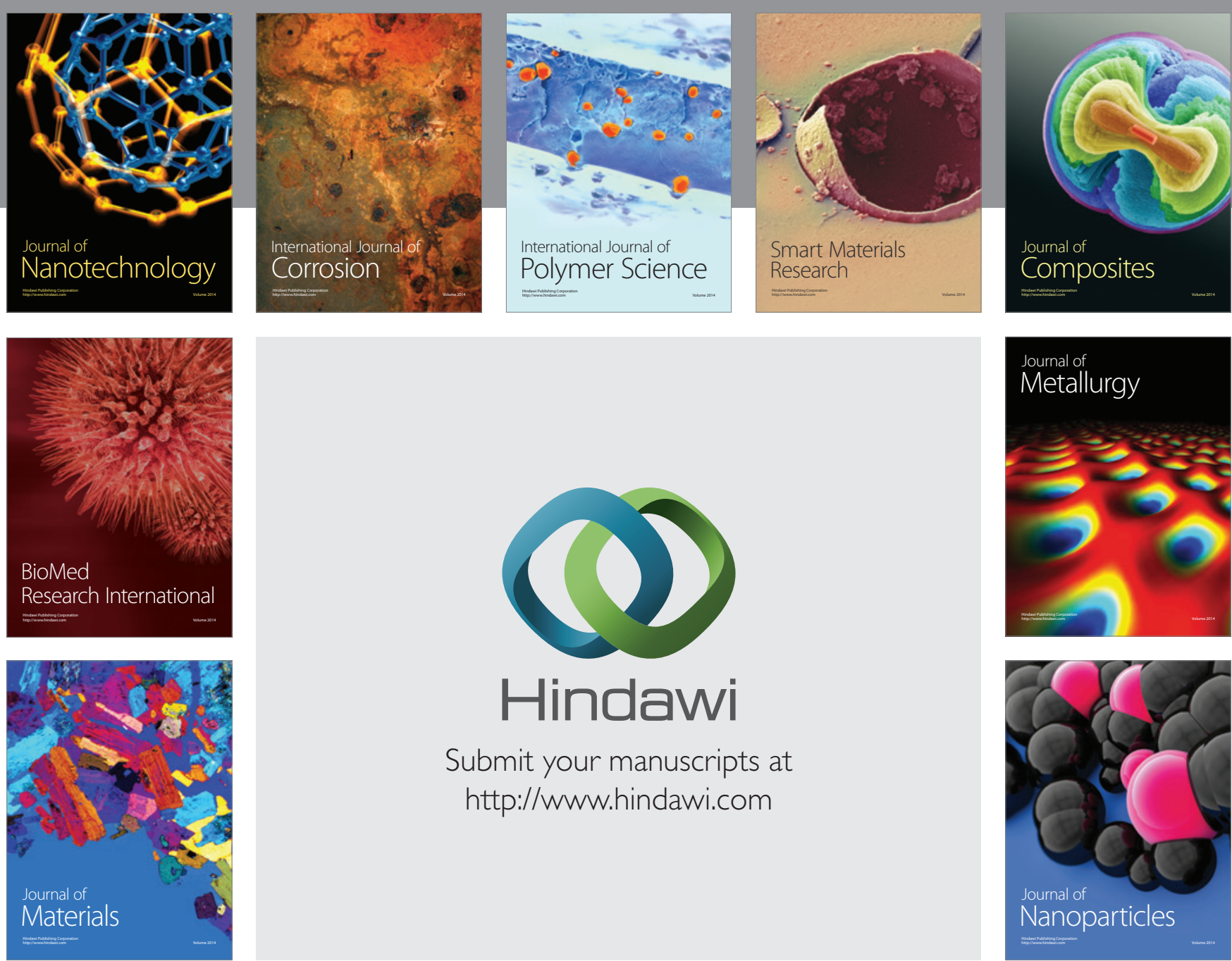

\section{Hindawi}

Submit your manuscripts at

http://www.hindawi.com

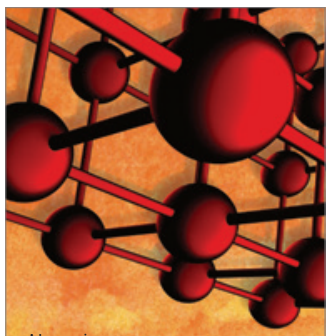

Materials Science and Engineering
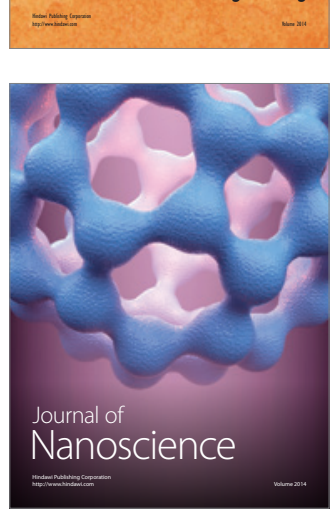
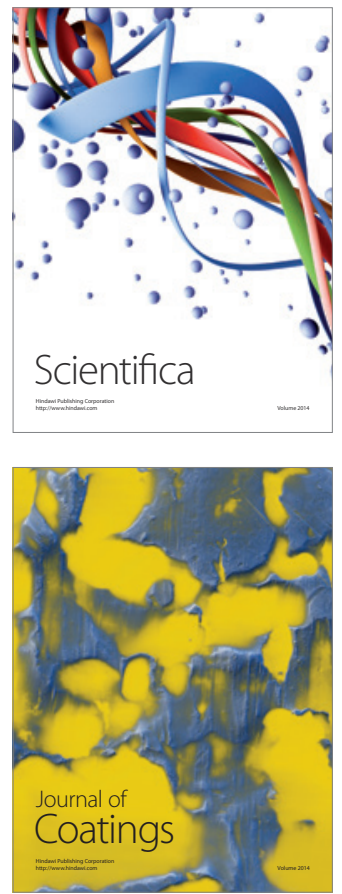
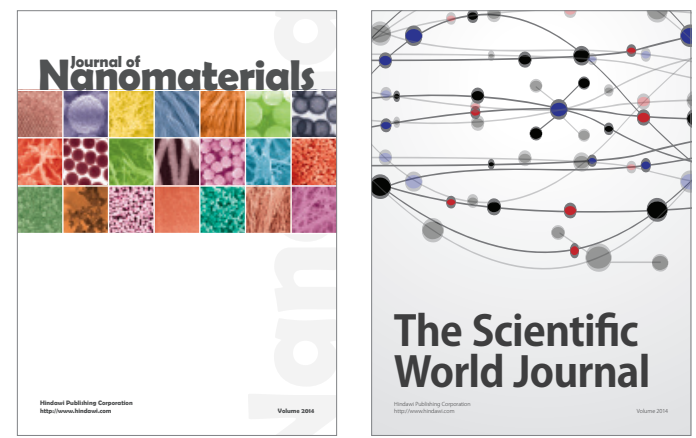

The Scientific World Journal
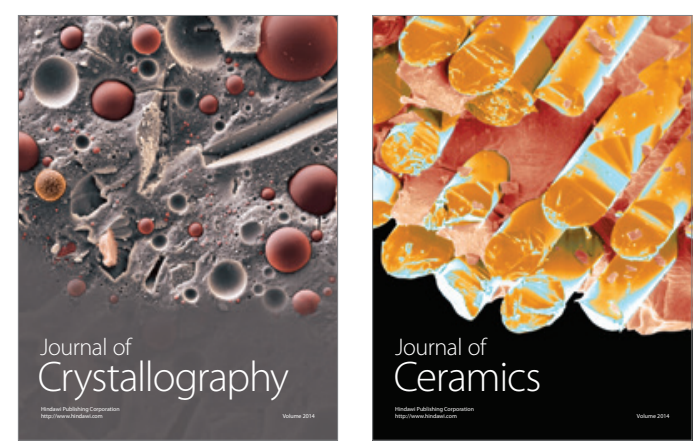
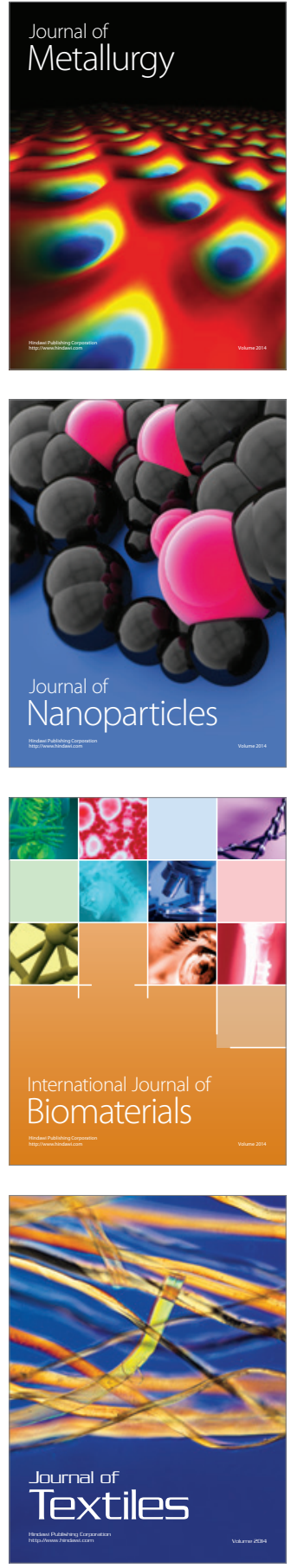